\title{
MULTIDIMENSIONAL FILTERING OF IRREGULARLY SAMPLED SEISMIC DATA
}

\author{
Ali Özbek and Ralf Ferber \\ Schlumberger Cambridge Research \\ High Cross, Madingley Road, CB3 0EL,Cambridge, UK \\ phone: + (44) 1223 325443, fax: + (44) 1223 361473, email: ozbek@cambridge.oilfield.slb.com \\ WesternGeco \\ Schlumberger House, Buckingham Gate, RH6 0NZ, Gatwick Airport, UK \\ phone: + (44) 1293 556858, fax: + (44) 1293 556800, email: ferber2@gatwick.westerngeco.slb.com
}

\begin{abstract}
In this paper, we introduce a new technique for multidimensional filtering of irregularly sampled seismic data. In this context filtering may be used for coherent noise and interference attenuation, as well as the generation of seismic images. The filtering operation consists of the convolution of the filter operator with the seismic data. The filter operator is usually computed on a regular grid (rectangular or hexagonal) that corresponds to the nominal sampling of the seismic data. Unfortunately, in the physical world the seismic data are often sampled at irregular spatial locations. Hence the two functions (the filter and the data) are defined on grids that do not match. Convolving these two functions without due regard to this fact would result in a degradation of the filtering performance. One way to solve this problem would be to interpolate the seismic data onto the regular grid. However, due to the shear volume of seismic data this approach would be prohibitively expensive in practice. We propose a methodology to solve the filtering problem in an accurate and economical way. In the proposed approach, the filter coefficients are first interpolated onto the irregular grid on which the seismic data are sampled. This is followed by the convolution of the filter operator and the seismic data over the common irregular grid. The convolution requires the numerical integration of the product of two irregularly sampled spatial functions, which can be performed by a $2 \mathrm{D}$ generalization of the trapezoidal rule. The numerical integration can be achieved by tessellating the irregular grid through Delaunay triangularization and computing geometry-dependent scaling factors for each grid point. The interpolated filter coefficients are then scaled by the geometry-dependent weights. The resultant filter coefficients can be optionally renormalized such that the filter response within a selected subset of the multidimensional spectrum is identical to that of the ideal filter. Finally, the modified filter can be applied to the seismic data using any conventional convolution procedure.
\end{abstract}

\section{INTRODUCTION}

Seismic data, in general, contain coherent and incoherent noise signals, along with seismic reflection signals. These noise signals interfere with the interpretation of the seismic signals and degrade the quality of the subsurface images that can be obtained by further processing. It is, therefore, very desirable to suppress the noise that is present in the recorded data before processing it for imaging. In land seismics, source-generated noise, like ground roll and airwaves, are the dominant noise types, and can lead to severe degradation in data quality. The conventional method of noise attenuation in seismic acquisition is through the process of analogue group forming. The analogue array output is the normalized sum (arithmetic average) of all input traces. The array support is usually rectangular. Consequently, the spectral response of the array is approximately a frequency-independent $2 \mathrm{D}$ sinc function in the kx-ky space. As the ground roll modes have finite apparent velocity, frequency independent filters are not ideal for separating signal and noise in seismic data. Highfrequency components of dipping seismic events may get attenuated, while the low-frequency components of the ground roll may be left in.

Point-receiver recording allows the use of digital group forming (DGF) for better noise attenuation and signal preservation. Digital group forming consists of the application of a 3D filter with two spatial and one temporal frequency axis, which allows much better control of the signal protection and noise rejection zones in the spectral (f-kx-ky) domain. Said 3D filter may be either deterministic [5], or adaptive ([3],[4). The natural data structures of the input data are individual common source gathers, e.g., the ensemble of all point-receiver recordings for an individual source position. On output, the data traces will be group formed, e.g., after convolution of the DGF filter with the point-receiver dataset. Usually, the number of output traces will be far less than the number of original point-receiver traces.

The design of multidimensional filters used for digital group forming conventionally requires regular sampling of the seismic data. Regular sampling in the context of this work means for example spatial sampling on a rectangular or a hexagonal grid, although other regular patterns can be used as well. Sampling of the time axis is usually regular, although the present 
work can be generalized to the case of irregular sampling in all dimensions involved.

In the physical world, the seismic data are often sampled at irregular spatial locations (Figure 1). This fact is usually ignored in the design and application of multidimensional filters for group forming. Consequently, the actual response can be different from the theoretical one. The signal pass zone of the filter in the spectral domain can be distorted, i.e., the pass zone may be smaller than designed, the gain may end up being significantly different than unity, and the phase significantly different from zero. The side lobes of the filter (i.e., the gain in the reject zone) may be significantly increased. This can lead to seismic signal distortion and noise leakage. Newman and Mahoney [2] studied the effects of errors in sensor positions in 1D linear arrays, and showed that the side lobes in the array reject regions are much higher than the theoretical values corresponding to the perfect arrays. We have made similar calculations for $2 \mathrm{D}$ and $3 \mathrm{D}$ arrays.

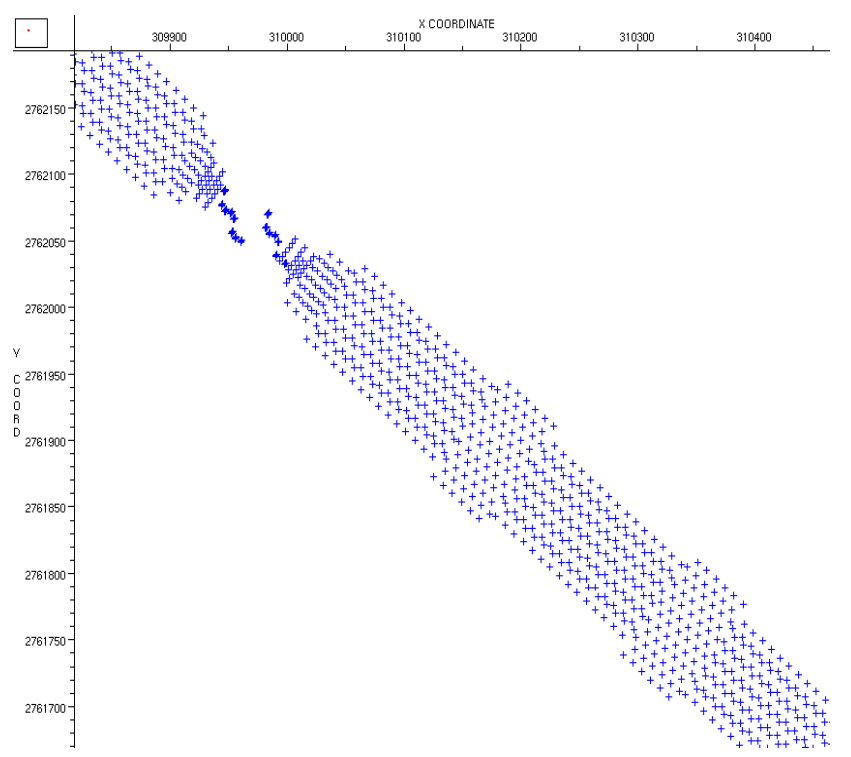

Figure 1: Example of receiver layout from a land survey.

One way to solve the problem of multidimensional filtering of irregularly sampled seismic data would be to interpolate (regularize) the irregularly sampled seismic data to a common regular sampling pattern, and apply filters designed for that regular pattern afterwards [1]. This approach would, in general, be too expensive, given the enormous volume of 3D seismic datasets. Furthermore, the spatial sampling irregularity in especially land acquisition often takes the form of the constriction of the array aperture, due to the obstacles (Figure 1). In these situations data regularization would have to take the form of extrapolation of severely aliased data, which is a numerical problem that one would prefer to avoid.

In this work, we discuss the design and application of multidimensional filters that are suitable for irregularly sampled seismic data without resorting to the regularization of the large volume of seismic data. As the size of the multidimensional filter operators is typically much smaller than that of the point-receiver data, the proposed technique is much cheaper than the regularization approach mentioned above. In addition, because the transfer functions of the filter operators are known to arbitrary accuracy, as opposed to the approximate knowledge of the frequency-wavenumber spectrum of the seismic data, the new approach has the potential to be more accurate as well.

\section{OVERVIEW OF THE METHOD}

Without loss of generality we assume that the temporal sampling of said seismic data is regular, although the method is also applicable to data irregularly sampled in time. In the following, we distinguish between ideal and actual data locations, to express the locations at which seismic data ideally should be collected versus the actual location at which data collection took place. Data collection means the process of actually recording seismic data at said locations, as well as the process of assigning seismic data spatial locations during further processing of the data, e.g., in the source-receiver midpoints domain.

Multidimensional filtering of irregularly sampled seismic data basically consists of 1) interpolation of the ideal filter given on a regular grid onto the irregularly distributed actual locations at which seismic data are gathered, 2) computing the weights of a multidimensional quadrature rule based on a tessalation of the actual data locations to evaluate the convolution integral, and 3) a normalization step to achieve identical filtering results within a selected subset of the frequencywavenumber domain. In detail, these steps are:

1. Design a multidimensional FIR (Finite Impulse Response) filter on a regular (rectangular or hexagonal, for example) grid. This grid may be optionally oversampled with respect to the ideal data locations.

2. Interpolate the filter onto the actual data locations to create new time-dependent filter coefficients for each actual data location. This can be done, for example, through sinc (band-limited), spline, polynomial, and other interpolation methods. Note, that the interpolation weights are the same for all the time lags of the filter. In preparation for the interpolation step the grid on which the original filter is defined should be extended, and the points outside the original support set to zero.

3. Compute the convolution integral using a multidimensional quadrature scheme, e.g., a 2D generalization of the trapezoidal rule. This can be achieved by assigning geometry-dependent weights to the actual data locations. The quadrature scheme requires a tessellation of the actual data locations, using, e.g., Delaunay triangularization. The geometry-dependent weights are proportional to the sum of areas of triangles for which a data location is a vertex.

4. Scale the interpolated time-dependent filter coefficients with the geometry-dependent weights.

5. Normalize the filter coefficients such that the filter response within a selected subset of the f-kx-ky spectrum is identical to that of the ideal filter. For seismic applications a useful such subset is along the frequency axis, i.e. $\mathrm{kx}=\mathrm{ky}=0$. For this particular case, 
the normalization rule is that for each time lag, the sum of the interpolated and geometry-dependent weighted filter coefficients equals the sum of the ideal filter coefficients.

6. Apply the modified filter using any multidimensional convolution procedure.

\section{EXAMPLE}

During the presentation, we will demonstrate the effectiveness of the new method using various real data examples. Figure 2 shows the receiver positions at a given point of the receiver line. It also shows the DGF spectral responses corresponding to the array with regular and irregular positions, as well as the corrected response corresponding to the irregular positions. The corrected response corresponds to the filter coefficients that are generated by the method introduced here. Not surprisingly, the corrected response cannot match the ideal response of the regular array, but both the pass region and the reject region responses have been significantly improved.

\section{DISCUSSION AND CONCLUSION}

The presented method of filtering irregularly sampled seismic data is an accurate, robust and economic alternative to regularizing the data onto a regular grid, followed by conventional filtering. In fact, in situations where the sampling is too distorted for the regularization to be numerically possible, filtering on the existing grid would be the only option. The method is applicable not only to noise filtering in land, marine or seabed seismic surveys, but to imaging as well.

Analyzing the contribution of the different components of the method has shown that, interpolation of filter coefficients is especially useful to correct the main lobe (pass zone) response. Geometry-dependent re-weighting of filter coefficients is especially useful to correct the side lobe (reject zone) response.

\section{AKNOWLEDGEMENT}

We would like to thank our colleagues Dave Arnold, Peter Blair, Charles Carvill, Mark Daly, Michael Kalkbrenner, John Quigley, Pete Welch and Wayne Wright for useful discussions and collaborations.

\section{REFERENCES}

[1] A. J. W. Duijndam, A.J.W., M. A. Schonewille, and C. O. H. Hindriks, "Irregular and Sparse Sampling in Exploration Seismology", in Nonuniform Sampling: Theory and Practice, ed. Marvasti, F., Kluwer Academic, New York, 2001

[2] P. Newman and J. Y. Mahoney, J.Y., "Patterns - with a pinch of salt". Geophysical Prospecting, v 21, pp.197-219, 1973

[3] A. Özbek, "Adaptive Beamforming with Generalized Linear Constraints", in SEG Conference Calgary, Extended Abstract SP 6.3, 2000a

[4] A. Özbek, "Multichannel Adaptive Interference Cancelling", in SEG Conference Calgary, Extended Abstract SP 6.5, $2000 \mathrm{~b}$

[5] A. Özbek, L. Hoteit, and G. Dumitru, "3-D Filter Design on a Hexagonal Grid for Point-receiver Land Acquisition", in EAGE Conference Paris, Extended Abstract, 2004. 

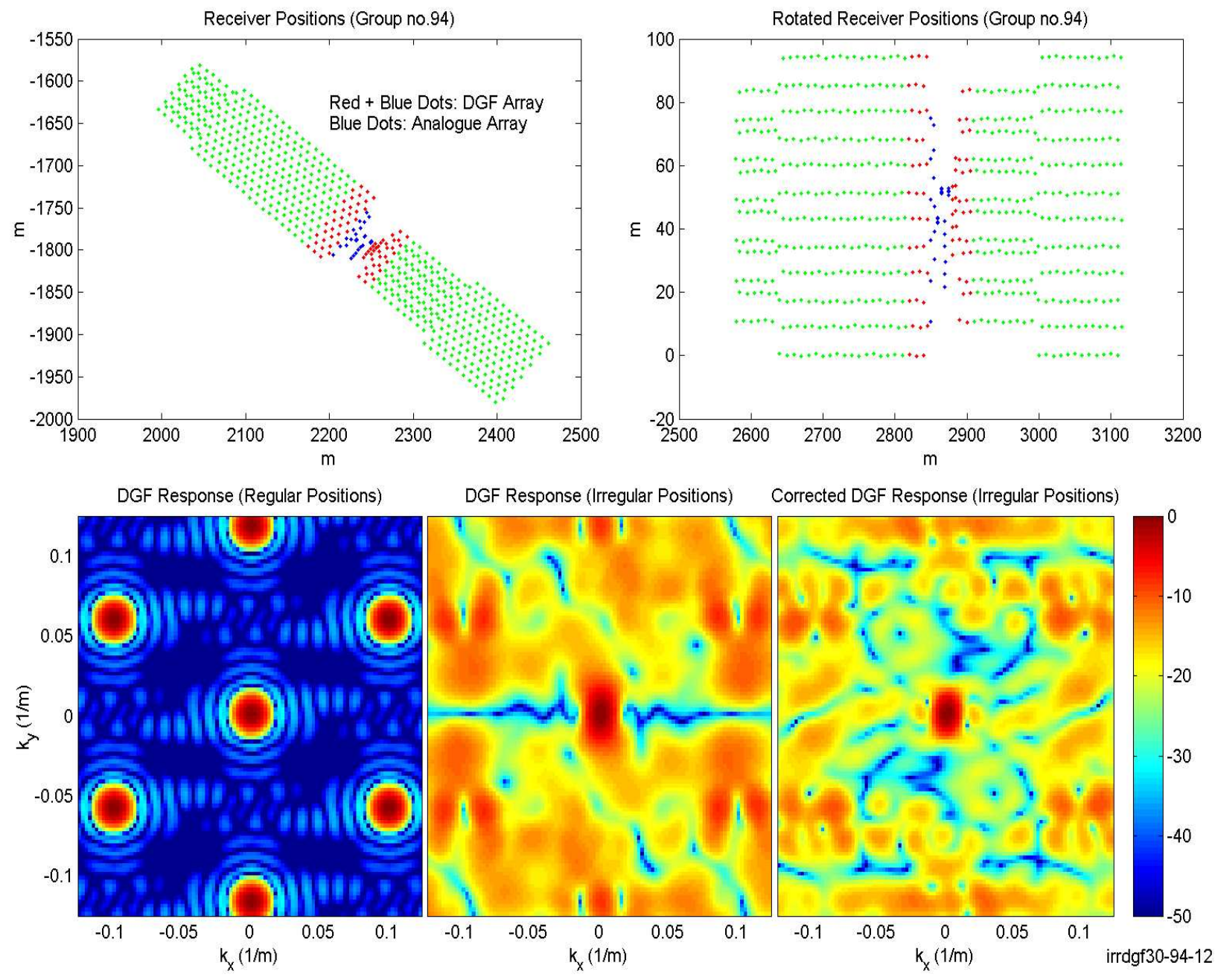

Figure 2: Receiver positions (top left), rotated receiver positions (top right), DGF spectral response corresponding to the regular positions (bottom left), response corresponding to the irregular positions (bottom middle), corrected response corresponding to the irregular positions (bottom right). 